\title{
L'eroe della tragedia. Pavese e il «Diario»
}

\author{
Giorgio BÀrberi SQUAROTTI \\ Università degli Studi di Torino \\ barberisquarotti@unito.it
}

\begin{abstract}
Riassunto
Questo saggio studia la struttura compiuta e definitiva al diario pavesiano, allo scopo di dimostrare come Il mestiere di vivere è offerto ai posteri come il risultato conclusivo di un'esperienza fondamentale quale è la scrittura. Il diario di Pavese si mostra come l'opera ultima e suprema dello scrittore, fino ad arrivare alla disfatta della vita e al trionfo della morte. Tramite il diario, lo scrittore diventa l'eroe indiscutibile della sua più tragica ed ultima opera esistenziale.
\end{abstract}

Palabras clave: Eroe, diario, scrittura, vita, morte, poesia, Pavese, Mestiere di vivere.

The hero of the tragedy. Pavese and the «Diary»

\begin{abstract}
This essay studies the perfect and definitive structure of the Pavesian diary to demostrate that Il mestiere di vivere offers to later generations the conclusive effect of the essential experience of Pavese: writing. The Pavesian diary revelas itself as the late and supreme work of Cesare Pavese, untill the end of his own life and the triumph of death. Thanks to his diary, Pavese becomes himself an unquestionable hero of his own most tragic and existential work.
\end{abstract}

Key words: Hero, diary, writing, life, death, poetry, Pavese, Mestiere di vivere.

Bàrberi Squarotti, Giorgio. 2011. L'eroe della tragedia. Pavese e il «Diario». Cuadernos de Filología Italiana, $\mathrm{n}^{\mathrm{o}}$ extraordinario: págs. 33-48.

A dire il vero, quello che più colpisce nel momento di affrontare $I l$ mestiere di vive$r e$ è la nota conclusiva che l'editore appone alla stampa del diario di Pavese: «Tutto questo fa schifo. Non parole. Un gesto. Non scriverò più» (Pavese 2000: 188).

Il diario di Pavese è stato trovato alla morte dell'autore tra le sue carte in una sbiadita cartella verde, su cui è scritto a matita rossa e blu: «Il mestiere di vivere di Cesare Pavese»: esso è costituito da fogli sciolti, numerati, manoscritti... a penna e 
qualche volta a matita, quasi sempre con cancellature e correzioni come in una prima stesura, e talora con correzioni a matita o d'inchiostro, evidentemente successive al tempo della stesura. Su una pagina bianca, messa da Pavese a mo' di frontespizio certamente negli ultimi suoi giorni di vita, è scritto: «1935-1950 Il mestiere di vivere di Cesare Pavese».

Sì, è un «diario», cioè, secondo il canone del genere, è il risultato della sequenza di annotazioni dello scrittore giorno per giorno, di vari argomenti, con l'indicazione delle date, che passano dalle confessioni del cuore alle esperienze di vita, dal commento delle letture agli appunti di scrittura, dagli incontri con amici e con donne amate $\mathrm{o}$ anche soltanto incontrate alle riflessioni di teoria letteraria, dai giudizi politici a quelli morali. Ma, al contrario dei «diari» a cui certamente Pavese guarda, per la sua lucidissima consapevolezza delle forme e delle ragioni dello scrivere e dei generi a cui dedicarsi nella sua vocazione letteraria - come lo Zibaldone leopardiano, il Diario intimo di Tommaseo, le Cento e cento e cento e cento pagine del libro segreto di D'Annunzio - c'è quel titolo, il nome dell'autore, la copertina, tutto quell'ordine di pagine che devono dare il senso dell'assoluta definitività dell'opera, là dove il «diario» è, necessariamente, il più tipico genere aperto e, al di là, non può non esserci altro ancora, altre notazioni o altre scritture d'altri generi, e toccherà ai posteri chiudere l'opera e darle la forma conclusiva. Il genere diaristico comporta il fatto che lo scrittore abbia, sul suo tavolo di lavoro o nell'ideale cartella che porta con sé ogni volta che esca di casa, il taccuino oppure i fogli pronti perché possa appuntare l'osservazione, l'emozione, l'idea, la lettura non appena gli venga in mente.

Ben prima di uccidersi, si direbbe che Pavese avesse già dato una struttura compiuta e definitiva al diario, trovando il titolo adeguato a quanto egli vuole che si legga, dopo la sua morte (o, almeno, la fine della sua scrittura, la decisione di non scrivere più, dopo che stabilito sia il libro, come i romanzi e come i dialoghi e come le poesie nelle loro variazioni narrative e liriche), di sé letterato e uomo. Non per nulla, allora, Il mestiere di vivere si conclude con una frase tanto drammatica ed esistenziale quanto letteraria, rivolta alla letteratura, non a un possibile interlocutore $o$ al lettore che spasimi e trionfi, soffra e comprenda come lui, secondo quanto il diario permette che si sappia: «Tutto questo fa schifo. Non parole. Un gesto. Non scriverò più» (Pavese 2000: 400; 18 agosto 1950). Pavese conclude nettamente e radicalmente con la dichiarazione che non vivrà più, cioè che ha deciso di uccidersi (di compiere, cioè, il gesto stoico, di cui tanto spesso parla fin dai primi anni di stesura del diario), ma con la proclamazione che non scriverà più nulla, nella letteratura che è specificamente sua, come sono la narrativa e la poesia e la critica, ma neppure nel diario, tanto aperto fino al giorno della scelta di far morire la parola scritta. La morte per suicidio è fatta coincidere da Pavese con l'uccidersi alla scrittura. Per questo $\mathrm{Il}$ mestiere di vivere è offerto ai posteri nella sua compiutezza, come il risultato conclusivo di un'esperienza fondamentale quale è la scrittura, che ha la vita come alternativa irrisolta (come accade per tanti altri autori a partire almeno da Rimbaud), e il diario viene a essere, per Pavese, l'opera ultima e suprema, quella appunto che offre esemplarmente l'esperienza fondamentale e autentica d'arte e di vita in gara e in lotta l'una con l'altra, alternamente ora vittoriose e perdute, fino alla disfatta della 
vita e al trionfo della morte. Lo scrittore Pavese vuole essere, nell'opera decisiva e conclusiva, come l'esempio più alto e strenuo della lotta sempre drammatica fra lo scrivere e il vivere: a malgrado della sublimità di quella scrittura (le poesie, i romanzi, i saggi, le novelle), a vincere non può che essere la vita, che coincide, allora, con la morte, in quella vocazione alla morte che è diventata, nel corso dei Novecento, nella storia come nella filosofia, l'alternativa all'élan vital, alla natura originaria e divina, agli dei della luce.

Poiché ancora le ultime battute del diario rappresentano la battaglia interiore fra la pazienza e la disperazione, la morte e la sopportazione anche della sconfitta più radicale, che è la perdita di Connie per la propria inettitudine, il fatto che $\mathrm{Il}$ mestiere di vivere venga consegnato ai posteri nella forma definitiva di opera compiuta per la stampa significa, a ben vedere, la vittoria della scrittura sulla vita sbagliata. 11 tragico su cui Pavese si è soffermato tanto a lungo fino al punto di scrivere un libro come i Dialoghi con Leucò - che sono la rappresentazione e il commento delle tragedie greche e del mito fino alla riscrittura, identificandosi ogni volta nei protagonisti, spiegando e, al tempo stesso, soffrendo fino in fondo tutti i loro errori, tutti i destini che gli dei hanno loro imposto, tutti gli strazi dell'anima e del corpo - viene a essere, ne Il mestiere di vivere, il compendio di tutte le esperienze dei miti. Non scrivere più, nell'ultima parola del diario, significa anche, con le pagine messe ben in ordine, la copertina, il titolo e il sottotitolo, un'ulteriore precisazione: dire, cioè, che la recita della tragedia esemplare è stata interamente compiuta nella battaglia fra la vita e la letteratura. La fine è eroica: il protagonista ha affrontato gli dei crudeli che gli hanno imposto un destino (l'inettitudine a vivere, ad amare nella forma piena e appassionata), l'ha sopportato, l'ha portato avanti fino a offrire come alternativa l'altro aspetto dell'esistenza, che è la scrittura, e il valore che egli è riuscito a fissare esemplarmente, imponendosi fra gli altri scrittori, i lettori, i giudici, come il migliore del suo tempo:

Sei consacrato dai grandi cerimonieri. Ti dicono: hai quarant'anni e ce l'hai fatta, sei il migliore della tua generazione, passerai alla storia, sei bizzarro e autentico... Sognavi altro a vent'anni? Ebbene? Non dirò 'tutto qui e adesso?' Sapevo quel che volevo e so qual che vale ora che l'ho. Non volevo soltanto questo. Volevo continuare, andar oltre, mangiarmi un'altra generazione, diventare perenne come una collina (Pavese 2000: 326).

È la notazione del 19 gennaio 1949. Pavese a questo punto si presenta come l'eroe vittorioso della scrittura. Così egli vince, al punto decisivo del suo itinerario dall'inizio della scrittura fino al successo e all'ulteriore trionfo che ancora si ripeterà: Pavese allora si proclama egli stesso un mito, come i protagonisti dei Dialoghi con Leucò. Si dice eterno come la collina delle Langhe dove tante volte ha incontrato gli dei, fatti anzi colline essi stessi, come la sua opera. La sequenza delle notazioni del diario, a questo punto, dopo la pubblicazione de La bella estate e il successo, registra via via la conseguenza di tale autocelebrazione: ma Pavese, proprio con l'incontro con Connie, si accorge di avere commesso la colpa di hybris. Nel diario, lucidamente e con qualche compiacenza, è troppo consapevole del significato di tutti i 
miti greci che portano inevitabilmente l'eroe vittorioso (nella lotta contro i mostri, nelle guerre, nello stesso scrivere: si pensi almeno ai dialoghi che hanno come protagonisti Saffo ed Esiodo) a essere esposto, a essere punito dagli dei per il suo orgoglio. In una delle ultime notazioni del diario Pavese corregge radicalmente la sua proclamazione di superbia citata prima, che coincide con la dichiarazione di consapevolezza di essere uguale agli dei, anzi a essi superiore. Dice: «La mia parte pubblica l'ho fatta - ciò che potevo. Ho lavorato, ho dato poesia agli uomini, ho condiviso le pene di molti» (Pavese 2000: 326; 16 agosto 1950). Sottilmente, Pavese, in queste parole, fa ammenda della sua hybris di eroe della letteratura, e piuttosto si confronta con altri poeti che non pretendono l'eccellenza e l'unicità, ma dicono umanamente che l'esito della loro attività di scrittori è l'aver offerto la bellezza della poesia agli uomini normali, comuni, e in questo modo hanno donato loro amore e pietà (e viene in mente, allora, il poema conviviale di Pascoli intitolato a Esiodo, il poeta degli iloti, non quello dell'epica, dei guerrieri, degli eroi: e nei Dialoghi con Leucò fra i protagonisti c'è anche Esiodo). Questo riconoscimento conclusivo, che Pavese pronuncia sul punto di morire (di uccidersi, come fanno gli eroi puniti dagli dei per la hybris di cui si sono resi colpevoli) è, appunto, analogo alle affermazioni degli eroi tragici: Edipo e Aiace come esempi fondamentali. È possibile (necessario) morire dopo aver riconosciuto che il destino è fatale e immutabile, ma anche dopo aver proclamato il valore e l'autenticità delle proprie opere: per quel che riguarda Pavese, dopo aver dato poesia agli uomini, cioè dopo aver scritto i libri che valgono a confortare, a illuminare, a far comprendere come è il mondo, la storia, soprattutto la vita contro cui si è battuto fin dalla giovinezza. L'uccidersi, allora, significa non già la verifica del fallimento, ma, al contrario, la giusta risposta di resistenza, di valore e di esemplarità al destino maligno e nemico.

A malgrado delle sue tare (del destino che ne è l'emblema), l'eroe intellettuale non cede alla disperazione, non chiede pietà, non supplica gli dei, rimanendo in vita, nella sua miseria, confessandosi vinto. Non agli dei che decidono il destino Pavese chiede grazia, ma piuttosto al Dio cristiano che ha patito fino alla morte, ed egli gli si sente vicino perché ha sofferto analogamente il tradimento, l'abbandono, la sconfitta della vita: «La cosa più segretamente temuta accade sempre. Scrivo: o Tu, abbi pietà. E poi?» (Pavese 2000: 400; 18 agosto 1950). L'interrogazione è proprio quella dell'uomo di fronte alla morte: se, appunto, ci sarà il nulla o ci saranno la pietà che gli varrà la preghiera suprema, oppure il giudizio della giustizia. Soffrire fino alla morte può essere stoico, cioè eroico secondo la filosofia antica, oppure può valere la misericordia. E tuttavia, Pavese «scrive» la sua preghiera d'invocazione, di pietà. Il diario trasforma sempre la vita (e la morte) in scrittura: per questo l'opera appare così compiuta e decisiva, senza nessuna possibilità di aggiungere qualcosa ancora, come al contrario è dello Zibaldone, del Diario intimo, delle Cento e cento e cento e cento pagine del Libro segreto di D'Annunzio tentato di morire. Se gli altri diari si arrestano casualmente, conservando sempre la possibilità di andare oltre, Pavese, invece, ha programmato rigorosamente la stesura della sua opera, fino alla fine della scrittura, che coincide con la scelta della morte.

Tutto è calcolato e ordinato. Il mestiere di vivere è il racconto di un'esistenza rappresentata come la lotta con l'angelo della parola e con il demonio della vita: il 
«mestiere», appunto, che è la scrittura, e il vivere che è amore perduto, fallimento, sofferenza, contraddizione di comportamenti, di azioni politiche, di scelte di lavoro, di amicizie, di decisioni morali. Nel diario la narrazione si svolge per blocchi contrapposti, per sussulti ciascuno distinto, che si possono nominare ora come «mestiere», cioè scrittura e letteratura, ora come «vita», e questa ha sempre in sé un nome specifico, che è quello di una donna: nel «mestiere» ci sono le considerazioni teoriche sul mito, e le prime osservazioni del diario vengono a essere quasi esclusivamente concettuali e riflessive; poi ci sono le notazioni critiche, d'interpretazione di autori cari allo scrittore, a partire dai narratori americani (Dos Passos, Anderson, Faulkner, ecc.), per passare ai classici, da Dante a Shakespeare e ai tragici elisabettiani, secondo i canoni poetici e narrativi con cui Pavese intende confrontarsi, alla ricerca dell'originalità necessaria, essendo la regola assoluta della letteratura del Novecento, che non può sopravvivere se non modifica la tradizione, nel momento stesso, magari, in cui usa e cita i modelli, le forme, le invenzioni dei classici. È significativo il fatto che Pavese si soffermi a lungo e rifletta sui classici a partire dalla guerra e dopo la guerra, mentre è impegnato nel maggiore sforzo creativo: compone romanzi su romanzi, scrive le sue operette morali con i Dialoghi con Leucò, che è, a giudicare da un altro punto di vista, il diario della propria vita e del proprio progetto narrativo, racconto e filosofia, teologia e lirica; ed è altrettanto importante il fatto che, nel diario, il discorso sul tragico greco e sulle sue riprese e modificazioni si faccia sempre più approfondito e impegnato quando il narratore cerca di uscire fuori da ogni forma di naturalismo e di materialità delle vicende e dei personaggi. Il tragico ne è quindi lo strumento, e le osservazioni su Shakespeare, su Marlowe, su Ben Jonson, su altri elisabettiani, sono in funzione di tale genere che Pavese vuole affrontare con strumenti narrativi, come dimostrano i tre romanzi brevi de La bella estate, i due de La casa in collina, fino a La luna e i falo, che è il tentativo supremo di rinnovare la tragedia classica con vicende, personaggi, rappresentazioni nuove.

Il tragico, a mano a mano che Pavese lo obiettiva nei romanzi, è rappresentato e discusso nel diario stesso. Ci sono sentenze fondamentali, che raccolgono l'intero programma pavesiano non in rapporto con la propria vita e neppure come teoria e commento sulla letteratura, ma come vero e proprio «titolo» e programma di scrittura, nella prospettiva di quel tragico a cui Pavese tende fin dagli anni 1939-1944. Nella notazione del 10 agosto 1944 egli riassume tante precedenti osservazioni, che varrà la pena di rivedere, dopo, e ricitare: «Nella tragedia greca tutto è sacro - cioè predetto, voluto da Dio»; a raffronto, si ricordi ancora l'ultima invocazione al «Tu», nell'ultimo giorno della scrittura del diario (Pavese 2000: 287).

$\mathrm{Ne}$ Il mestiere di vivere si rivela tanto spesso nel modo della riflessione dei loghia, dei giudizi, l'aspirazione di Pavese all'autentica tragedia del suo tempo, in contrapposizione al romanzo borghese dell'età romantica, che egli identifica esemplarmente in Guerra e pace di Tolstoj, e soprattutto alle sue conseguenze peggiori, che sono il romanzo naturalista e quello descrittivo. Dice Pavese nell'intervento del 16 giugno 1940:

La natura astrattamente descrittiva di Giganti di Doëblin rivela che Berlin-Alexaderplatz anche là dove pareva farcita di esperienza umana e di meditazione era soltanto materiata di brutta e banale verità quotidiana, descritta non drammatiz- 
zata; che è difetto comune a molta narrativa attuale e della tua. Doëblin, Dos Passos, tu: se volete sfuggire al verismo epidemico, cascate nell'astratta costruzione espressionistica. Vi manca soprattutto il senso del dramma (Pavese 2000: 188).

Bisogna arrivare fino al tragico, e proprio in questa prospettiva si devono leggere Paesi tuoi e, subito dopo, i Dialoghi con Leucò, che sono, allora, da considerarsi non come la reinvenzione, in tempi moderni, dei miti antichi, partendo da Leopardi ma anche da Pascoli (così raramente ricordato quando si parla delle riflessioni e della scrittura del mito a proposito di Pavese, e, invece, i miti dei Dialoghi con Leucò hanno argomenti analoghi ai Poemi conviviali), quanto piuttosto come il punto di partenza necessario per salire fino al dramma, cioè alla tragedia. Nella notazione del 15 ottobre 1940 c'è interamente in nuce non soltanto l'aspirazione al tragico nella narrativa, ma anche il senso de $\mathrm{Il}$ mestiere di vivere come strenua e appassionata tensione al supremo significato della scrittura pavesiana fino alla conseguenza conclusiva, che è l'ultima affermazione del 18 agosto 1950, ed è la tragedia.

L'intera vicenda dell'autore del diario ripercorre abbastanza visibilmente l'itinerario di una ricerca letteraria e di un'esperienza della vita per giungere alla suprema ambizione della rappresentazione dell'eroe tragico. Si legga la notazione del 15 ottobre 1940: «La sola regola eroica: essere soli soli soli. Quando passerai una giornata senza presupporre né implicare in nessun tuo gesto o pensiero la presenza di altri, potrai dirti eroico. O altrimenti essere Cristo - cioè annientarsi. Ma l'hai detto ieri - nessuno rinuncia a ciò che conosce - e tu conosci troppe cose» (Pavese 2000: 205).

La conclusione de $I l$ mestiere di vivere fin da questo passo fa pensare a una tensione ancora più strenua e ambiziosa: nel diario scrivere la tragedia come congiungimento del tragico greco e del tragico cristiano, l'eroe che si erge contro gli dei, a malgrado del destino, testimoniando con la sua solitudine l'autenticità e il valore esemplare, e l'eroe che si umilia fino alla morte in croce, in questo modo opposto tuttavia rilevando la sua assoluta significazione che non può avere confronto, perché è ugualmente sublime e unica. È ulteriormente significativo il fatto che a un certo punto della notazione Pavese si rivolga a se stesso: egli si presenta come l'eroe della tragedia greca e come l'imitatore di Cristo, nell'opposta ma ugualmente fondamentale decisione della solitudine esemplare, anzi, meglio, ideale, come ha da essere il protagonista della nuova e grande letteratura del suo tempo; ed egli è, appunto, prima di tutto se stesso, poi oggettivandosi nelle poesie (Lavorare stanca, su cui Pavese tante volte ritorna, prima e dopo la prima edizione dell'opera, anche a qualche distanza, quando già la scelta del genere è la narrativa) e nei romanzi, e, in modo più compiuto onde mettere insieme narraziome, riflessione, critica e lirica, nei Dialoghi con Leucò, che sono l'altra faccia de Il mestiere di vivere.

Sono, allora, opportune altre citazioni, sempre in funzione del chiarimento del concetto di tragico in relaziome con tutte le altre scritture. Dice Pavese, in data 26 settembre 1942: "La situazione tragica greca è: ciò che deve essere sia. Di qui il meraviglioso dei numi che fanno accadere ciò che vogliono; di qui le norme magiche, i tabù o i destini, che devono essere osservati; di qui la catarsi finale che è l'accettazione del dover essere» (Pavese 2000: 245). 
Le ultime notazioni nella conclusione del diario sono mirabilmente vicine a quelle di un decennio prima o poco dopo:

Cara, forse tu sei davvero la migliore - quella vera. Ma non ho più il tempo di dirtelo, di fartelo sapere - e poi, se anche potessi, resta la prova, la prova, il fallimento. Vedo oggi chiaramemte che dal 28 a oggi ho sempre vissuto sotto quest'ombra... Eppure, anche tu sei soltanto un pretesto. La colpa, dopo che mia, è soltanto dell'inquieta angosciosa, che sorride da sola (Pavese 2000: 398-399; 16 agosto 1950).

La prova e il fallimento sono gli altri nomi del destino dell'eroe, del protagonista della letteratura sublime che si concreta nella tragedia greca (e cristiana). Egli deve necessariamente avere un nome nella vita come tutti coloro che lo conducono alla morte, con i vari accidenti che lo sollecitano a capire l'ineluttabilità della sua sorte, in questo estremo caso «tu», forse la migliore e la vera, ma proprio per questo la causa finale della scelta di battersi con la Morte e perire (la Morte che sorride nel punto di affrontarla una volta per tutte). Dice ancora Pavese nella notazione del 17 agosto 1950:

Ho ignorato per qualche anno le mie tare, ho vissuto come se non esistessero. Sono stato stoico. Era eroismo? No, non ho fatto fatica. E poi, al primo assalto dell' 'inquieta angosciosa', sono ricaduto nella sabbia mobile. Da marzo mi ci dibatto. Non importano i nomi. Sono altro che nomi di fortuna, nomi casuali - se non quelli, altri? Resta che ora so quel che è il mio più alto trionfo - e a questo trionfo manca la carne, manca il sangue, manca la vita (Pavese 2000: 399-400).

Poco prima è detto: «Nel mio mestiere... sono re». È un'espressione singolare, e Pavese l'usa a ragion veduta. Come eroe intellettuale è «re», ma il re che, come Edipo, affronta il fallimento, il destino avverso, l'inganno degli dei, come è stato quello dello scrittore, che è arrivato più in alto di tutti, al potere supremo della scrittura, e che proprio in quel punto incontra imprevedutamente l'inciampo di «lei» che gli fa riconoscere il suo destino, come, appunto, Edipo che si trova davanti a Tiresia (come racconta Pavese ne La strada, nei Dialoghi con Leuco).

Il re non può che affrontare il suo destino: nella penultima notazione del diario Pavese carica all'estremo di significato le sue affermazioni, le sue sentenze. In quanto re, Pavese è consapevole del suo potere, ma anche della sua fragilità umana, in quanto ha nascosto per tanti anni il suo destino, proprio con il fasto della regalità della sua arte a cui tutti i contemporanei si inginocchiano. Il destino, dopo che Pavese ha verificato con drammatica essenzialità - anche fortemente enfatica per i tre verbi che ripetono «manca» - il proprio fallimento, comporta «un gesto», quello che deve fare l'eroe-re sconfitto nel punto stesso del trionfo più alto. Pavese non scrive più, perché la tragedia, che coincide con la sua letteratura e con la sua vita, è finita, ed egli lo sa bene, dopo aver commentato e interpretato il tragico greco, Sofocle, Eschilo, Euripide, e poi Shakespeare, gli Elisabettiani, la vicenda di Cristo. Può chiamarla Il mestiere di vivere e metterla bene in ordine, per la lettura e la stampa e tutte le repliche delle rappresentazioni, delle letture e dei commenti (e, infatti, quan- 
to si è scritto e detto e pianto sul vivere e sul morire di Pavese), come dimostra il modo con cui l'editore l'ha trovato e, subito, l'ha pubblicato. L'esaltazione conclusiva della sconfitta tragica dell'eroe-re allude ancora una volta alla doppia faccia dell'esperienza di Pavese: la scrittura e la vita. Come accade a Edipo, che giunge al sommo trionfo del regnare, sposare la regina vedova, generare figli, anche Pavese racconta e raffigura il suo trionfo di scrittore; ma ugualmente l'uno e l'altro urtano contro il destino e scelgono il gesto decisivo dell'eroica e stoica autodistruzione (è quasi insignificante il fatto che Edipo si acciechi e che Pavese si uccida).

La differenza sta nel fatto che Pavese rappresenta il proprio destino come quello dello scrittore migliore del suo tempo e, al tempo stesso, disperatamente inetto, impotente, incapace di vivere, di mordere nella carne, nel sangue, cioè nella vita. Pavese non protesta davanti agli dei quando parla del suo fallimento della vita: come dice, quando riflette sulla tragedia greca, essa comporta la fatalità degli eventi, delle azioni, delle conseguenze, e la stessa hybris dell'uomo (dell'eroe, del «re») è inevitabile.

Scrivere Il mestiere di vivere come la grande tragedia moderna, del tutto libera da naturalismo, realismo, accumulazione di personaggi e di eventi, pateticità romantica, viene a essere l'ambizione suprema per Pavese. Per di più, scriverla è possibile soltanto a patto di farla diventare definitiva, perché, dopo, l'eroe non potrà e non dovrà più né scrivere né vivere, entrambe le esperienze tuttavia attuandole e sperimentandole fino in fondo, cioè dopo tanti anni di sconfitte e altrettanti anni di successivi trionfi, finché arte e vita giungono al punto di coincidere e di attuare così il tragico moderno più alto dove mai la tragedia né greca né elisabettiana è riuscita ad arrivare. Si comprende in questo modo la struttura de $\mathrm{Il}$ mestiere di vivere: al contrario del Diario di Tommaseo e dello Zibaldone, l'opera di Pavese è abbastanza chiaramente prevista e calcolata fin dal momento in cui lo scrittore decide di affrontare questo genere di scrittura, tanto è vero che dal 6 ottobre 1935 fino al 18 agosto 1950 Pavese non interrompe mai la stesura del diario, se mai occasionalmente moltiplicando o rallentando le notazioni (al contrario di quanto hanno fatto gli altri «diaristi»), ma sempre misurando e organizzando il suo diario in vista della pubblicazione, quando ormai sono venuti meno sia lo slancio lirico ed epico, sia la costruzione narrativa, neppure pensando a chiuderlo se non con la decisione di non scrivere più. Il protagonista delle Cento e cento pagine ha, come sigla esemplare, il nome di Cocles, che si dichiara tentato di morire, come, del resto, frequentissimamente dice Pavese nel corso della stesura de Il mestiere di vivere. Ma D'Annunzio vuole meglio rilevare il suo intento di aggiungere agli altri generi fino a quel momento sperimentati quello ulteriore del diario, mescolato di vita e di letteratura, d'arte e di sesso e disperazione. L'impostazione del diario dannunziano influisce certamente nella scelta del genere diaristico da parte di Pavese, che ha già scritto racconti, almeno un romanzo, le poesie che, in parte, verranno a costituire Lavorare stanca, ma, dopo D'Annunzio - con tutte le novità e le contraddizioni della scrittura a cominciare dalle avvanguardie e dopo la Prima Guerra Mondiale -, Pavese, per la lucidissima capacità critica e teorica che lo distingue, sa di dovere andare oltre e altrove rispetto a Döblin, a Dos Passos, ad Anderson, a Hemingway ecc. Per questo il suo diario è così rigorosamente pro- 
grammato, là dove quello stesso di D'Annunzio lascia spazio alla memoria, al ricupero di pagine o di versi «antichi», alle rievocazioni della guerra, che sono sostanzialmente al di fuori del canone del genere.

Pavese ne Il mestiere di vivere offre una continuità sicura e precisa. Il tempo non ha né intervalli né ritorni indietro o indugi nella memoria. Se accenna all'infanzia, è soltanto in funzione dell'arricchimento ulteriore del «mito», e per questo cita allora Vico e Leopardi. A Pavese non interessa per nulla la commemorazione dell'infanzia, che è oggetto di tanta narrativa degli anni fra la Prima e la Seconda Guerra Mondiale. La memoria è, invece, il grande tema di D'Annunzio narratore de $I l$ secondo amante di Lucrezia Buti e de Il compagno dagli occhi senza cigli. Per Pavese la memoria autentica, quella moderna, da rappresentare sia in versi sia nella narrativa, è quella del mito antico, e in questa prospettiva il diario viene a essere, al tempo stesso, di immediata registrazione della vita e di trasferimento di essa in sentenza, in giudizio; in trasfigurazione nelle figure e nelle vicende dei protagonisti dei miti originari. La continuità de $\mathrm{Il}$ mestiere di vivere è data dalla trasposizione delle figure e delle esperienze della vita in canone letterario, anche quando il diario registra i nomi delle donne amate o conosciute, quelli degli amici e dei colleghi, i paesi, le poche città, i particolari di vigneti, di rive, di conche d'acqua, di torrenti, di colline delle Langhe. Pavese insiste tante volte sulla necessità di non descrivere, perché, in questo caso, si cade nel naturalismo, che è l'opposto della rappresentazione moderna, in versi e in prosa. Dice Pavese, in data 22 settembre 1938:

\begin{abstract}
Basta a volte, nella seconda riga, una pennellata naturalistica ('Faceva un tempo fresco, con un po' di nebbia') per provocare pagine e pagine di naturalismo implacabile, documentarie e non più narrate, dove cioè ogni avvenimento si colloca sul piano della detta pennellata, rifiutando di lasciarsi ripensare... Il vero racconto (Primo amore e il Campo di grano) tratta il tempo come materia non come limite e lo domina scorciandolo e rallentandolo e non tollera didascalie che sono il tempo e la visione della vita della vita reale; piuttosto, risolve in impulso (sintesi fondamentale o idea generatrice) di costruzione (distanza prospettica o ripensamento) l'ambiente temporale (Pavese 2000: 118-119).
\end{abstract}

1119 novembre 1939, per lo sviluppo ulteriore delle considerazioni sul rifiuto del naturalismo in quanto scelta della descrizione che si pretende obiettiva, Pavese parla dell' «immagine-racconto» che istituisce il simbolo, e chiarisce in seguito il concetto parlando di Dante (26 novembre 1939): «Dante sul Purgatorio non si volta mai a contemplare il panorama, per la ragione che non descrive realisticamente un viaggio, ma espone un simbolo dove si ricorre alla scena, al visibile, solo in quanto si veste di corpo un concetto. Non ha quindi obblighi di rispettare la logica naturalistica del reale» (Pavese 2000: 163).

Il 10 dicembre 1939 Pavese sentenzia: «Non più simbolo allegorico, ma simbolo immaginoso» (Pavese 2000: 165). Il 9 marzo 1940 ritorna sulla contrapposizione fra il naturalismo e la narrazione «mitica» che egli vuole attuare:

Il naturalismo ha insegnato ai narratori... che nulla che non sia azione deve entrare nel discorso. Allora si descriveva l'ambiente che era parte dell'azione, e gli 
eventi, oggettivamente; ora tutto ciò si descrive guardando con l'occhio del personaggio; ma è per tutti acquisito che non si deve più digradire. Come nel naturalismo l'autore doveva scomparire davanti alla realtà, così ora esso deve scomparire davanti all'occhio del personaggio (Pavese 2000: 165).

Le citazioni possono essere moltiplicate: ma deve essere chiaro che, in ogni caso (ed è il carattere unico, straordinario de Il mestiere di vivere), Pavese parla della sua narrativa, delle poesie di Lavorare stanca, ma altresì delle notazioni del suo diario in quanto genere «nuovo» in assoluto, non descrittivo, non documentario, soprattutto quando parlano di sé, delle sue esperienze, degli incontri, delle donne amate, fino alla sentenza, all'aforisma, che è il modo più efficace e scarno per evitare il documento, la realtà vissuta, il fatto singolo, la persona com'è. Anche in questo caso Pavese vuole giungere al significato, all'esemplarità, al «mito», che è, poi, il risultato estremo della sua ricerca letteraria che è totalitaria: citazione, modello e intervento della vita riportata alla parola.

Si pensi, a confronto, alle evocazioni sontuosamente immaginose delle donne di D'Annunzio nelle Cento e cento pagine (un esempio perfetto è quello di Helène Zancle), addirittura inventate al di là di tutte le possibili descrizioni e in questo modo libere da ogni documentarietà, e d'altro canto alle donne del diario di Pavese: il nome, l'esperienza d'amore e disamore, l'ingiuria, la considerazione sul tradimento o sul fallimento, ma senza nessun concretamento fisico che consenta di fissarla in figura, in persona della vera vita. Fin dalle prime notazioni, gli amori, le sconfitte, gli incontri nel diario non devono diventare carne, sangue, vita. La formula tipica è l'accusa o l'autoaccusa, ed entrambe diventano immediatamente il giudizio definitivo, che deve giungere fino all'aforisma che vale una volta per tutte. Pavese cerca di vincere la vita con la forma della scrittura più astratta, assoluta, sintetica proprio nel momento in cui registra le vicende quotidiane. Ma allora si capiscono le tante osservazioni e i tanti commenti sul naturalismo negativo, sul simbolo, sul mito: anche il diario va composto in tali modi.

A ben vedere, i Dialoghi con Leucò sono la suprema conquista della non descrizione, del non documento, della non presentazione di sé e delle persone con cui Pavese viene a trovarsi in rapporto e degli amori e della stessa storia, per quel poco che egli cita (e ancora meno negli anni della guerra, in tale caso con qualche breve notazione e sempre trasferita nel commento, nel giudizio morale). A proposito dei bombardamenti su Torino, Pavese osserva le reazioni, dopo, non della gente, ma del pensiero dello scrittore, che guarda soltanto al concetto come esempio assoluto. E la notazione del 16 giugno 1940, quando ci sono stati i primi bombardamenti sulla città:

(Allarmi aerei) Gli stridori, i tonfi, gli scoppi, che fanno trasalire tutti in questi giorni, non solo prima della guerra non spaventavano, essendo innocui, ma non erano nemmeno percepiti. Ogni passione - qui, il terrore - crea una particolare sensibilità verso i propri stimoli e pretesti, e rivela tutta una provincia della vita oggettiva che prima passava innosservata. L'uomo più oceanico è quello 'trasmutabile per tutte guise'. Finchè si avranno passioni non si cesserà di scoprire il mondo (Pavese 2000: 188). 
La citazione dantesca è molto rivelativa: il terrore degli uomini sotto i bombardamenti è riportato al concetto universale, alla condizione umana com'è in assoluto, in quanto è compendiata nell'esperienza che Dante fa nello stato degli uomini dopo la morte, per i tre regni della dannazione, della purgazione e della beatitudine. L'uomo può mutare continuamente, perchè continuamente si trova di fronte a eventi, esperienze, novità della vita: ma quello che conta è il fatto che lo scrittore è in grado di comprendere, di giudicare, di definire di che si tratta, come il terrone sotto i bombardamenti, che valgono, per Pavese, come strumento per la scoperta ulteriore del mondo. Il terrore della guerra viene riportato al nuovo esempio del vivere, a confronto con la precedente vicenda innocua fatta di rumori e fragori normali, senza che comportino il pensiero della distruzione e della morte pur sempre incombente.

Il diario di Pavese non è mai «intimo», ma concettuale, mitico. Nel trascorrere degli anni esso viene a precisarsi sempre meglio nella vita che vuole diventare concetto e riflessione e per questo si confronta inevitabilmente con la morte. Si pensi, nei Dialoghi con Leucò, a Schiuma d'onda, dove parlano Saffo e Britomarti, l'una che ha scelto la morte buttandosi nel mare di Grecia, l'altra una ninfa dei monti e delle rupi, che si è trasformata in onda per sfuggire al mortale che voleva afferrarla e possederla $\mathrm{e}$, in questo modo, togliere la sua libertà, la sua capacità di mutare continuamente. Sono le due figure della doppia condizione della scrittura: la vita e la poesia, e proprio in questa prospettiva Pavese racconta la vicenda opposta della poetessa di Lesbo e della ninfa, entrambe diventate schiuma d'onda, perché svanire è la suprema aspirazione, l'annullamento e la metamorfosi, fino a diventare uguali. E il modo di affrontare la vita che comporta la sconfitta e la morte (il suicidio), trasfigurandola nella tragedia che, tuttavia, si compie non per l'eterna pace, ma per l'angoscia che dura ancora, il destino non accettato (per quel che riguarda Saffo, mentre Britomarti è il suo destino: si pensi alle ultime notazioni del diario pavesiane, quelle dell'identificazione conclusiva fra fallimento eroico di fronte alla vita e fine della scrittura: anche Saffo non «canta» più, nella decisione di diventare eroina tragica; e dietro, allora, c'è L'ultimo canto di Saffo di Leopardi).

I Dialoghi con Leucò sono una continua rappresentazione del tragico moderno per il tramite della trasposizione nei nomi dei miti «antichi», riportati da Pavese nell'attualità, e specificamente nella propria vicenda, in questo modo sublimata e resa esemplare, concretando in eventi mitici i concetti e le sentenze e gli aforismi ai quali egli riduce i fatti (gli amori, la guerra, gli amici, le donne sempre necessariamente irraggiungibili, fino alla reazione di ripudio di sé, fino alla vocazione alla morte). I personaggi dei miti si trovano di fronte al destino, agli dei, alla morte, eroica o disperata, in ogni caso sempre esemplare, mai lamentabile e neppure accettata.

Penso, in particolare, a La Madre, che ha come protagonista Meleagro, fatto morire dalla madre in uno scatto d'ira. Meleagro dice a Ermete: «O Ermete, tutto ciò è il mio destino. Ma son pure esistiti mortali che vissero a sazietà senza che nessuno avesse in pugno i loro giorni», ed Ermete risponde:

Tu ne conosci, Meleagro? Sarebbero dei. Qualche vile è riuscito a nascondere il capo, ma anche lui portava sangue di madre, e allora l'odio, la passione, la furia sono divampati nel suo cuore solo. In qualche sera della vita anche lui si è senti- 
to riardere. Non tutti - è vero - siete morti di questo. Tutti, quando sapete, conducete una vita di morti (Pavese 2000: 322).

Il riardere dell'uomo che non ha misurati i suoi giorni fin dall'inizio, per quanto vile, non si salva dalla morte. Ermete parla a Meleagro, ma Meleagro è il narratore stesso, il protagonista del diario. Pavese sa (e Meleagro ne è l'emblema mitico) che, come già gli è accaduto, incontrerà la fiamma che lo arderà: la donna della crisi di prima, la donna futura che inevitabilmente, secondo il suo destino, incontrerà, e sarà la fine. Pavese è stoico, cioè guarda all'altra alternativa, che è il tragico, in quanto affronterà eroicamente il suo destino, senza rimpianti e senza proteste, al contrario di Meleagro, che dice: «Ma nemmeno vedere i miei figli... non conoscere quasi il mio letto...»; ed Ermete ribatte: «Hai avuto fortuna. I tuoi figli non nasceranno. Il tuo letto è deserto. I tuoi compagni vanno a caccia come quando non c'eri. Tu sei un'ombra e il nulla». È la prefigurazione del destino di cui fin dalle origini Pavese è consapevole, e sceglie il dialogo narrativo e filosofico - come le Operette morali di Leopardi - per farselo dire, come in quasi tutti i Dialoghi con Leucò, dal dio che tutto sa e decide il destino e assiste senza intervenire mai (e ne è esempio appunto Ermete).

Il diario è l'attuazione, nel mondo ormai senza dei e senza il sacro, del destino degli uomini: c'è un legame strettissimo fra Il mestiere di vivere e i Dialoghi con Leucò, nel senso che questi appaiono, in gran parte, come l'obiettivazione dell'esperienza dello scrittore nelle sue attività, nelle sue riflessioni, nelle sue angosce, nei suoi dolori, nei suoi fallimenti di strazio e di morte, dai quali può uscire soltanto l'eroe che tutto ha capito e che accetta di affrontare la stoica tragedia, dopo aver attraversato le origini del mondo, della vita, dei mostri, del sangue, degli dei. Ma l'eroe tragico dei tempi moderni non è un cacciatore come Melagro, né un re come Edipo, né qualche semidio come Prometeo oppure Eracle: è come Esiodo il poeta, colui che è in grado di offrire bellezza e verità agli uomini e, infatti, Mnemosine spiega al poeta della Teogonia quale è il suo compito, oltre che venerare la dea della memoria: raccontare le origini del mondo, degli dei, della vita. Dice Mnemosine:

Non capisci che l'uomo, ogni uomo, nasce in quella palude di sangue? E che il sacro e il divino accompagnano anche voi, dentro il letto, nel campo, davanti alla fiamma? Ogni gesto che fate ripetete un modello divino. Giorno e notte, non avete un istante, nemmeno il più futile, che non sgorghi dal silenzio delle origini (Pavese 2000: 165).

Esiodo si sente schiacciato dalla lezione della dea dell'Elicona: «Tu parli, Melete, e non posso resisterti. Bastasse almeno venerarti». Mnemosine dice allora al poeta: «C'è un altro modo, mio caro... Prova a dire ai mortali queste cose che sai». Pavese alla fine del diario spiega quello che egli - l'Esiodo moderno, il poeta d'oggi, il migliore della sua generazione - ha fatto: «Ho lavorato, ho dato poesia agli uomini, ho condiviso le pene di molti». Ha, cioè, obbedito alla lezione di Mnemosine. Quello che ha detto agli uomini è, appunto, la verità delle origini di sangue e fango, da cui, però, nascono il sacro e il divino, che lo scrittore, al contrario degli 
autori del tempo attuale, riesce a raffigurare nel momento stesso in cui li incarna nei personaggi dei suoi romanzi e nei dialoghi con le divinità antiche.

Per questo Pavese tanto spesso nomina le colline, le Langhe, la gente del paese ne Il mestiere di vivere: egli ha sempre di fronte gli emblemi delle origini sacrali, e le vicende della sua esistenza come quelle dei suoi personaggi non sono altro che la ripetizione delle rovine, dei fallimenti, delle morti, delle pene che gli dei hanno fissato come destino di ciascun uomo, nell'ambito supremo del tragico che si identifica con la condizione umana. Il privilegio di Esiodo/Pavese è di essere consapevole del sangue e del fango da cui nascono gli dei e gli uomimi e, quindi, di avere di fronte, in sé e in tutti i personaggi che crea nella sua sorte di poeta, insieme con il dovere di dire, anche la scelta del tragico, che è il culmine dell'esperienza esistenziale e della scrittura. Le donne amate, soprattutto, vengono a essere, ne Il mestiere di vive$r e$, la prova del proprio dovere di scrivere come alternativa all'amare e al vivere e, al tempo stesso, dell'inganno che deve essere affrontato fino in fondo, e che comporta l'atto eroico, cioè il tragico, il sacrificio di sé, la morte. Si comprende, allora, ulteriormente il processo così lucido e determinato della registrazione pavesiana delle notizie biografiche. Le donne amate - in particolare quella che ha la voce roca e quella che è venuta dalla primavera e dal mare - sono evocate in modo così scarno perché suscitano, sì, il dolore, il furore, lo sconforto, la disperazione, ma sono rappresentate come le prove ogni volta decisive della scrittura che costantemente aspira all'estrema coincidenza con la verità (non con la realtà) della vita.

La maggior parte dei Dialoghi con Leucò per il tramite dei miti antichi rappresenta vicende di sacrifici cruenti, di uccisioni, di suicidi, di sfide agli dei e di sconfitta, di inganni, e sangue, fango, sperma, fiamma, acque, violenze, ne sono la causa e l'effetto; e i romanzi pavesiani tutto questo trasferiscono nelle Langhe, a Monticello, a Santo Stefano Belbo, nelle colline, a Torino, non soltanto per un'effettiva attenzione a quei paesaggi e a quei paesi e a quella città, ma soprattutto perché così è possibile concretare, al di là di ogni forma di realisticità e di naturalismo, la verità del destino e, di conseguenza, del tragico. Gli aforismi, i giudizi, le accuse generali contro le donne, non sono certamente la conseguenza di una misoginia banale e neppure la pura e semplice registrazione della vita, bensì il costante impegno di Pavese nel cercare di renderla concetto, sentenza, astrazione, principio generale, in funzione della scrittura. Lo stesso accade, significativamente, per le osservazioni sulla guerra e successivamente sul dopoguerra, con le considerazioni su amici, conoscenti, altri letterati, politici, donne e uomini degli incontri romani, poi dei successi letterari, fino all'affrettato fervore dei premi, delle recensioni, della conoscenza con Connie. Pavese non dice altro che nomi, o commenti rapidissimi, rapidissimi giudi$\mathrm{zi}$, tanto è vero che il suo diario non offre mai un quadro della storia, la condizione di una società, la prospettiva di un costume letterario. Se mai, tutto questo è trasportato in un altro spazio, che è quello della scrittura. Si pensi esemplarmente al nulla o quasi che Pavese dice ne Il mestiere di vivere degli anni decisivi 1943-1945: la guerra civile, la Resistenza, sono altrove, ne La casa in collina e ne La luna e $i$ falò, trasportati infatti nell'esemplarità del tragico, del tutto al di fuori della quotidianità delle esperienze personali e delle motivazioni della cronaca. 
La guerra e gli scontri cruenti della Resistemza sono tradotti in mito e in tragedia nei Dialoghi con Leucò, ma, in questo caso, l'astrazione è ancora maggiore, in forza dei nomi dei miti e dei loro destini esemplari, validi una volta per tutte, per cui il compito dello scrittore è quello di raccontarli e spiegarli, per poi riportarli nelle attuali ambientazioni più efficaci, come le Langhe e Torino. Il diario per questo lascia il maggiore spazio alle considerazioni teoriche della lettetatura, del genere, che a Pavese sta più profondamente a cuore, cioè il tragico. L'eroe stoico, di cui Pavese parla nel diario, in realtà si trasforma sempre più chiaramente e lucidamente nell'eroe tragico, quello del mito antico (greco), e Pavese chiarisce il concetto soprattutto nei Dialoghi con Leucò, con il conforto dei Poemi conviviali pascoliani forse ben prima di Nietzsche e di D'Annunzio (e per quest'ultimo il discorso vale soprattutto per Fedra e per la Laus vitae, ma non senza la citazione de La morte del cervo, con l'apparizione del centauro e degli altri miti estivi della Versilia: Niobe, la palude, il sangue, Dioniso, Pasifae, Arianna, Icaro e Creta). Pavese, nel diario, si fa autore dei miti e attore, narratore e partecipe, e può così far risorgere gli dei e il sacro, fino al punto di condurre al compimento la più esemplare vicenda dell'eroe tragico dei tempi modermi, poeta e drammaticamente partecipe della vita, ma soltanto in funzione della scrittura della più compiuta tragedia del mondo attuale; e, in questo, accetta di morire, così inverando in modo assoluto il significato, il valore, l'esemplarità della sua opera (e in questa prospettiva si leggano i giudizi su Vittorini, che finisce a essere presentato come il precursore, l'unico vero nella letteratura contemporanea, in preparazione dell'esaltazione suprema della propria arte).

L'inconsolabile, nei Dialoghi con Leucò, ha come protagonista Orfeo e la baccante Bacca. Dice Orfeo:

$\mathrm{Fu}$ un vero passato soltanto nel canto. L'Ade vide se stesso soltanto ascoltandosi. Già salendo il sentiero quel passato svaniva, si faceva ricordo, sapeva di notte. Quando mi giunse il primo barlume di cielo, trasalii come un ragazzo, felice e incredulo, trasalii per me solo, per il mondo dei vivi. La stagione che avevo cercato era là, in quel barlume. Non m'importò nulla di lei che mi seguiva. Il mio passato fu il chiarore, fu il canto e il mattino. E mi voltai (Pavese 2000: 178).

Orfeo precisa ancora che, scendendo nell'Ade, ha compiuto il suo destino, cantando secondo i suoi modi la vita e la morte. Pavese, in questo modo, si identifica con Orfeo, proprio nel «dialogo» del cantore e della baccante, per la sua arte della vita e della morte (delle sue poesie, dei suoi racconti, dei suoi romanzi, anche in previsione di quelli che scriverà, fino alla discesa definitiva nell'Ade, ma in quanto eroe tragico che è, al tempo stesso, cantore esemplare, sublime, come, appunto, Orfeo). Bacca dice conclusivamente a Orfeo: «Forse è per questo che ti seguono, le donne della Tracia. Tu sei per loro come il dio. Sei disceso dai monti. Canti versi di amore e di morte». E'come se Pavese volesse a questo punto prefigurare, insieme con il divino della sua scrittura, anche l'inevitabile destino: essere dilaniato dalle donne che pure hanno preso il poeta come un dio. Bacca sa bene che le donne si infatuano per il poeta, ma, poi, l'abbandonano e, anzi, accettano di straziarlo, emblematicamente, non fisicamente nei tempi attuali, e sempre in modo inevitabile ripetono così 
il mito: le sentenze di Pavese sulle donne amate e sulle donne in generale, così aspre e sprezzanti, vengono a essere, allusivamente e drammaticamente, la ripetizione della vicenda di Orfeo, cioè del poeta esemplare, quello che per primo offre il suo canto agli uomini e agli dei. Pavese viene accolto e respinto, ammirato e abbandonato, ma è anche vero che il poeta è andato troppo oltre, canta l'amore e la morte (come la donna di Eresso in Solon, il primo dei poemi conviviali di Pascoli), davanti agli dei dell'abisso.

Pavese nel diario dice di voler mostrare, nelle esperienze e nelle vicende della vita, l'esemplarità ideale dei protagonisti del mito, e proprio per questo, a un certo punto, pone al centro della sua scrittura i Dialoghi con Leucò e alla fine Il mestiere di vivere. Fin dalle prime notazioni egli rileva che le «favole antiche» non sono morte, come lamenta Leopardi, e per poterlo dimostrare accetta di attraversare anche gli orrori dei miti e dei fallimenti, le angosce, le disperazioni quanto Orfeo ed Esiodo. Egli le riode perché le ridica. È un'aspirazione che conduce alla consapevolezza della propria sconfitta esistenziale: il diario ne è la dimostrazione. Nell'ultimo dei Dialoghi con Leucò, che si intitola Gli dei, in cui non ci sono più interlocutori con i nomi dei miti, dice Pavese che sulle colline delle Langhe gli abitanti hanno visto gli dei.

- Mi domando sù è vero che li hanno veduti. - Chi puo dirlo? Ma sì, li han veduti. Han raccontato i loro nomi e niente più - è tutta qui la differenza tra le favole e il vero, 'Fu il tale o il tal altro'. 'Ha fatto questo, ha detto quello'. Chi è veritiero, si accontenta. Non sospetta nemmeno che potranno non credergli. I mentitori siamo noi che non abbiamo mai veduto queste cose, eppure sappiamo per filo e per segno di che mantello era il centauro o il colore dei grappoli d'uva sull'aia d'Icario (Pavese 2000: 169).

Il primo dei due che parlano sul monte rievocando coloro che incontrarono gli dei e citando il loro nomi che li hanno uditi è consapevole che è ormai impossibile rivederli, perché le cose oggi sono quello che sono, realisticamente, e non hanno più nomi divini, ma quelli scientifici o dati dai dizionari degli uomini che non sanno più nulla degli dei. Egli dice: «Questi luoghi hanno nomi per sempre... quel che è stato, è stato per sempre. Io, ti assicuro, ti ho ascoltato come riascolto dentro me quei nomi antichi». L'ambizione suprema di Pavese è, allora, quella di risalire sulle colline, ripronunciare i nomi dei luoghi come quelli pronunciati dagli dei che gli uomini antichi hanno incontrato.

Il «dialogo» è tutto intriso di citazioni leopardiane: Della primavera delle favole antiche, l'Inno ai patriarchi, l'Ultimo canto di Saffo (ma anche dei Poemi conviviali pascoliani: Solon, Alexandros, I vecchi di Chio, Psyche, ecc.). Ma Pavese sale a ritrovare le favole antiche - a malgrado di quel rischio mortale che comporta sulle Langhe, che non sono uno spazio puramente geografico, quanto quello della vita vera e della tragedia moderna, quando egli ne parla ne $\mathrm{Il}$ mestiere di vivere e nei romanzi, l'unico oggi possibile per vivere e scrivere. Dice il primo degli interlocutori del dialogo de Gli dei: «Io, per me, non mi stanco di sentirli parlare dei loro terrori notturni e delle cose in cui speravano». Pavese è giunto da un altro spazio e sa 
molto bene di non appartenere alla gente che era salita sulle Langhe per incontrare gli dei, ma vuole parlare con chi ha visto gli dei, ascoltando i loro nomi. È la spiegazione più appassionata e drammatica della scrittura narrativa di Pavese, ma anche del diario. È l'aspirazione a ritrovare gli dei, al prezzo di esserne folgorati; e per questo è inevitabile affrontare il fallimento della vita, soprattutto l'esperienza che, altrimenti, salverebbe, cioè l'amore, la generazione. Chiede l'altro interlocutore de Gli dei: «E credi ai mostri, credi ai corpi bestiali, ai sassi vivi, ai sorrisi divini, alle parole che annientano?» Le vicende raccontate nei romanzi e vissute o commentate o giudicate nel diario sono la trasposizione attuale dei miti: hanno l'analogo significato di onore, di eticità, di scelta della morte, di fronte al supremo trionfo della parola e alla sconfitta ugualmente suprema dell'esistenza.

A cominciare dal diario, quando Pavese meglio concreta il suo programma poetico e narrativo, dopo Ciau Masino e i primi racconti e accanto a Lavorare stanca, l'aspirazione è di incontrare di nuovo gli dei e rinnovare le favole antiche, con tutto l'orrore e la tragicità che comporta, ma anche con la certezza di ritrovare gli dei, pur tremendi e crudeli quali sono (e il suicidio ne sarà la dimostrazione). Dice, conclusivamente, il primo interlocutore de Gli dei:

Credo in ciò che ogni uomo ha sperato e patito. Se un tempo salirono su queste alture di sassi o cercarono paludi mortali sotto il cielo, fu perché ci trovavano qualcosa che noi non sappiamo. Non era il pane né il piacere né la cara salute. Queste cose si sa dove stanno. Non qui. E noi che viviamo lontano lungo il mare o nei campi, l'altra cosa l'abbiamo perduta.... quei loro incontri (Pavese 2000: 170-171).

Pavese vuole ritrovare gli dei, per quanto mortale sia il pericolo che comporta. Per questo sale sulle colline, nel diario come in Paesi tuoi, fino allo scontro decisivo e tragico ne La luna e i falò. Non impunemente si fanno risuscitare le favole antiche e si cerca di incontrare di nuovo gli dei, ma Pavese pensa di essere l'unico capace di farlo poiché se ne crede degno per la sua scrittura, al prezzo di perdersi nella vita. L'incontro con gli dei si attua nel momento in cui Pavese accetta di morire sprezzando la vita e chiudendo la sua vicenda di scrittura. Il mestiere di vivere ha questo significato, proprio nel momento in cui si propone come il libro conclusivo e davvero compiuto come compendio di tutte le poesie e i romanzi e i racconti.

\section{Bibliografia}

PAVESE, Cesare (2000): Il mestiere di vivere. Diario 1935-1950. Torino, Einaudi. PAVESE, Cesare (2006): Dialoghi con Leucò. Torino, Einaudi. 